\title{
Fisiopatologia do traumatismo raquimedular. Uma revisão
}

\author{
Sergio Henrique do Amaral', Marcelo Nery Silva', Marcelo Barletta Soares \\ Viterbo', Mauricio Giraldi', Clemente Augusto de Brito Pereira ${ }^{3}$
}

Serviço de Neurocirurgia do Hospital Heliópolis, São Paulo, SP, Brasil

\section{RESUMO}

A compreensão dos mecanismos fisiopatológicos envolvidos no traumatismo raquimedular é essencial para possibilitar uma mudança no prognóstico atual do paciente com lesão medular. É apresentada, neste artigo, uma revisão dos mecanismos fisiopatológicos da lesão medular traumática.

\section{PALAVRAS-CHAVE}

Traumatismos da medula espinhal. Fisiopatologia.

\section{ABSTRACT}

Physiolopathology of the spinal cord trauma. A review.

The understanding of the pathophysiological mechanisms of the spinal cord trauma is essential to make possible a change in the present prognosis of patients with spinal cord injury. It is presented a review of the pathophysiological mechanisms of the traumatic spinal cord injury.

\section{KEY WORDS}

Spinal cord injuries. Physiopathology.

\section{Introdução}

O traumatismo raquimedular (TRM) é uma entidade nosológica altamente incapacitante. Freqüentemente, pacientes acometidos por tal lesão apresentam restrição funcional que impossibilita o retorno às atividades usuais, tanto profissionais quanto lúdicas.

Nos Estados Unidos é estimada a ocorrência de mais de 12 mil lesões medulares por ano, o que corresponde a uma incidência anual de 11,5 novos casos por milhão de habitantes. A maioria desses casos ocorre devido à lesão decorrente de projétil de arma de fogo ou em virtude de acidentes automobilísticos $8,24,33,35$.

No Brasil, a incidência anual estimada de lesão medular decorrente de trauma é de 22,6 casos novos por milhão de habitantes. As causas principais são as quedas, notadamente as de laje, e o mergulho em água $\operatorname{rasa}^{7,9,16}$.
O paciente com lesão medular tem alta incidência de morbidades associadas, além de internações múltiplas e prolongadas, com uma taxa de mortalidade de $4,4 \%$ a $16,7 \%$ somente no período da primeira internação ${ }^{23,34,55}$.

Fica claro o alto impacto social e psicológico dessa doença, tanto nos pacientes como nos seus familiares, além da repercussão financeira nos diversos sistemas de saúde (público e privado) para tratar e reabilitar tais pacientes. Estima-se um gasto anual de US\$ 4 bilhões por ano nos Estados Unidos com o tratamento da lesão medular traumática e suas conseqüências ${ }^{50}$. Sendo assim, o traumatismo raquimedular tem enorme impacto na qualidade de vida do paciente e na sociedade.

É necessário, então, promover mecanismos adequados para prevenir a ocorrência de trauma raquimedular e para tratar de forma mais eficiente os pacientes com lesão medular, o que só será possível por meio de um amplo conhecimento da sua fisiopatologia ${ }^{14,17}$.

1 Neurocirurgião, médico-assistente do Serviço de Neurocirurgia do Hospital Heliópolis, São Paulo, SP. 


\section{Histórico}

O primeiro relato escrito de lesão medular traumática provém de papiros do Egito Antigo, os papiros de Edwin Smith. Nesse relato as lesões medulares eram consideradas intratáveis ${ }^{12,27}$. Muitos escritos posteriores também consideravam tal lesão intratável e essa foi a conduta adotada nos pacientes com TRM por séculos. No final do século XIX, uma série de estudos começou a elucidar a fisiopatologia do TRM e a sugerir, pela primeira vez, uma possibilidade de terapia para os pacientes com lesão medular traumática. Uma breve descrição das contribuições mais importantes se faz necessária.

Em 1890, Schmaus ${ }^{45}$ publicou um estudo que evidencia alterações patológicas em medula de coelho após trauma e descreveu o surgimento de degeneração e cavitação central na medula.

Em 1911, Allen ${ }^{2,3}$ investigou, em cães, o papel da necrose hemorrágica e da hematomielia na lesão medular, sendo o primeiro autor a sugerir a existência de uma lesão medular secundária, o que iniciou uma nova fase na compreensão da fisiopatologia do TRM.

Já Riddoch ${ }^{44}$, em 1917, sugeriu a possibilidade de reparo e regeneração medular após trauma.

Em 1954, Tarlov e Klinger ${ }^{53}$ demonstraram que o alívio precoce da compressão medular resulta em melhora neurológica.

E Richardson e cols. ${ }^{42,43}$, nos anos 1979 e 1980 , demonstraram, em ratos, regeneração de axônios medulares com o uso de enxerto de nervo periférico.

Todos esses autores, assim como muitos outros não citados aqui, dedicaram-se ao estudo da fisiopatologia do TRM por acreditar que existe uma chance de evitar a progressão e recuperar o dano neurológico decorrente da lesão medular e que isso só poderá ser alcançado quando conseguirmos responder à pergunta: $\mathrm{O}$ que acontece após o trauma?

\section{Fisiopatologia do TRM}

A fisiopatologia da lesão medular traumática envolve a compreensão de dois mecanismos, ou momentos, de lesão: a primária e a secundária ${ }^{4,21,49}$.

\section{Lesão primária}

A lesão primária é a resultante do trauma mecânico inicial. Pode ser compreendida como a combinação do impacto inicial com uma eventual compressão subseqüente. Tem relação direta e imediata com o trauma e, uma vez que ocorre, é irreversível. O dano medular direto é resultante da transferência da energia cinética para a medula espinhal e pode ocorrer por meio de quatro mecanismos básicos: o estiramento, a laceração, a compressão e a secção $0^{56}$. Tal trauma determina dano nos axônios, nas células gliais e nos vasos sangüíneos, em diferentes graus (parcial ou completa).

O único meio de evitar a lesão primária é impedir a ocorrência do trauma, o que, por sua vez, só pode ser obtido com políticas de educação e conscientização da população, diminuindo, assim, a incidência de tais lesões.

\section{Lesão secundária}

A lesão secundária é aquela que segue a primária e a sua fisiopatologia envolve múltiplos mecanismos. Todos ocorrem de forma concomitante e estão relacionados entre si, potencializando uns aos outros, de modo que não é possível afirmar qual é o mecanismo fisiopatológico principal28,39. São eles (Quadro 1):

- mecanismos vasculares;

- mecanismos iônicos;

- mecanismos bioquímicos;

- mecanismos inflamatórios;

- mecanismos celulares.

Por ocorrer após o trauma, o dano secundário é passível de terapêutica sendo, então, potencialmente reversível. A compreensão de sua fisiopatologia irá possibilitar, em algum momento, o tratamento da lesão medular (Quadro 2).

\section{Mecanismos vasculares}

A isquemia medular é o mecanismo vascular per se. Ocorre uma diminuição significativa do fluxo sangüíneo medular logo após o trauma, que piora nas primeiras horas e perdura por ao menos 24 horas $^{32,54}$, sendo determinada por uma combinação de fatores locais e sistêmicos. Pode ser seguida por uma fase de hiperemia ou "perfusão de luxo" em decorrência de uma redução do $\mathrm{pH}$ perivascular causada pelo acúmulo de metabólitos ácidos, como o lactato. Essa reperfusão tecidual aumenta a lesão celular, pois promove o influxo local de radicais livres e outras substâncias tóxicas ${ }^{4}$.

Entre os fatores locais envolvidos com o surgimento e a manutenção da isquemia medular, destacam-se: o vasoespasmo secundário ao dano mecânico, a produção de eicosanóides, a trombose (via agregação plaquetária), o estresse oxidativo, a perda da auto-regulação vascular e, com destacada importância, a hemorragia na substância cinzenta medular e o edema vasogênico ${ }^{11}$. 


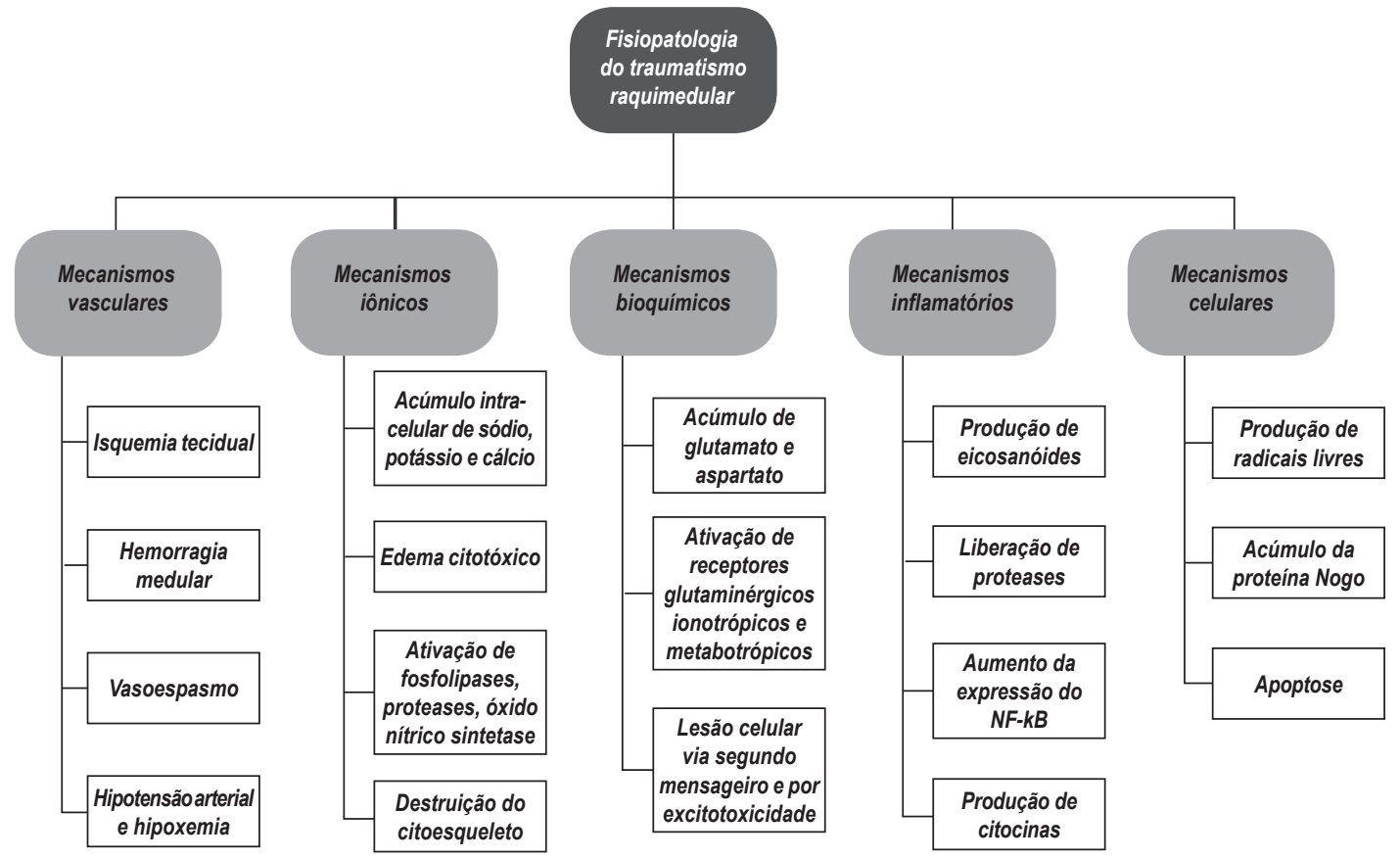

Quadro 1 - Fluxograma contendo os mecanismos de lesão secundária envolvidos na fisiopatologia do traumatismo raquimedular.

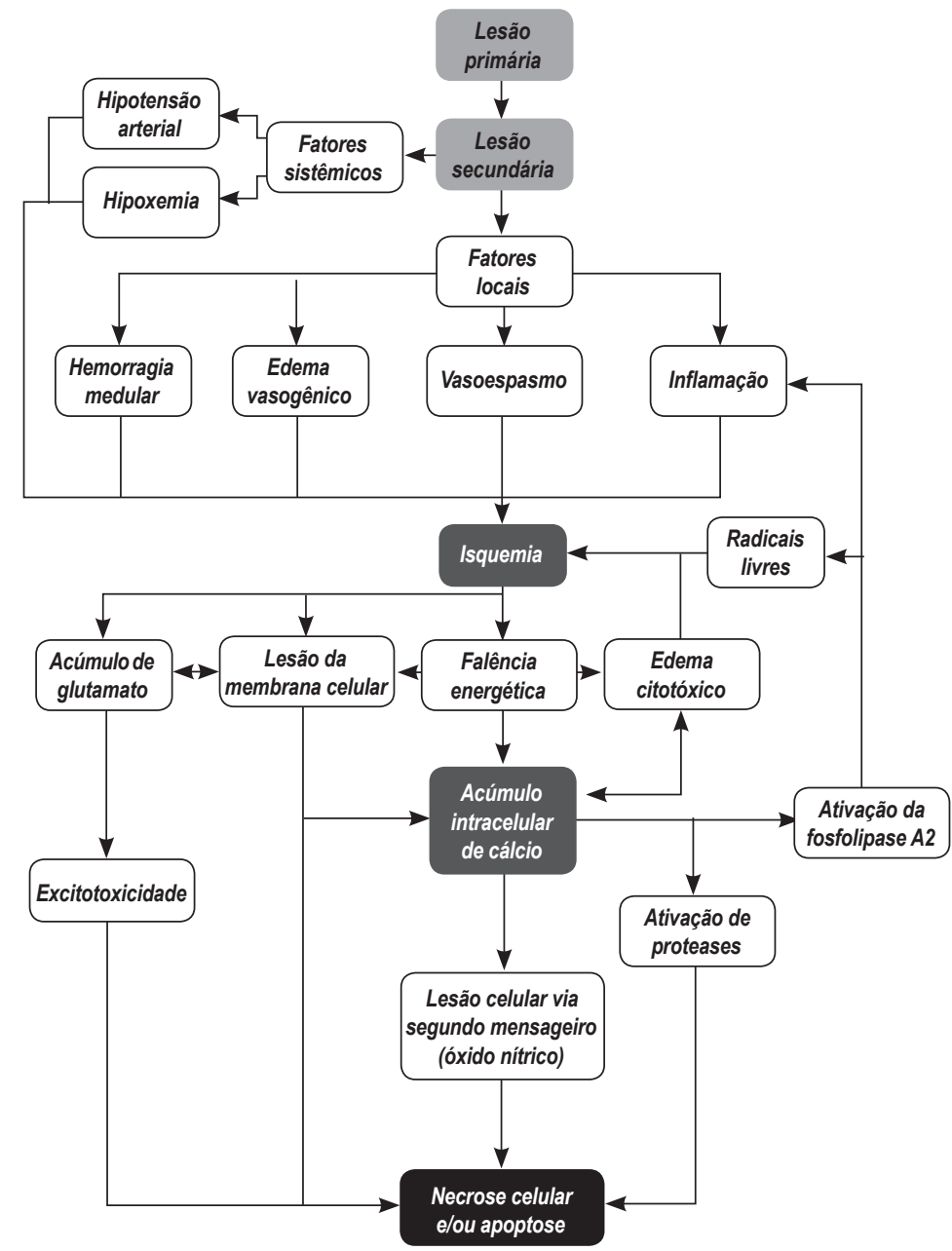

Quadro 2 - Fluxograma contendo sumário da fisiopatologia do traumatismo raquimedular. 
A hemorragia na substância cinzenta surge nos primeiros cinco minutos após o trauma e determina uma diminuição da perfusão local, que fica praticamente ausente durante a primeira hora. Acomete inicialmente a porção central da medula e, progressivamente, por expansão do hematoma, atinge a substância branca. Subseqüentemente, ocorrem necrose hemorrágica central (após horas) e posterior mielomalácia (após semanas) ${ }^{57}$.

O edema vasogênico ocorre em virtude de lesão endotelial secundária ao trauma, que determina aumento da permeabilidade vascular. Surge algumas horas após o trauma e pode perdurar por até 15 dias. Está relacionado com o surgimento da necrose (não hemorrágica) na substância branca ${ }^{26,61}$.

O principal fator sistêmico é a hipotensão arterial sistêmica. Esta pode ser causada por diversos mecanismos, tais como o choque cardiogênico, o hipovolêmico e o neurogênico. O choque neurogênico é aquele determinado pela diminuição do tônus simpático, ocasionado pela lesão medular, com conseqüente diminuição da resistência periférica (vasodilatação periférica) e do débito cardíaco, da bradicardia e da hipotensão arterial $^{18}$. Acredita-se que uma pressão arterial média de $85 \mathrm{mmHg}$ seja suficiente para evitar a contribuição desse fator sistêmico à isquemia medular.

Outro fator é a hipoxemia secundária à insuficiência respiratória, muitas vezes ocasionada por lesão medular cervical alta ${ }^{59}$.

\section{Mecanismos iônicos}

Os principais íons envolvidos na fisiopatologia da lesão medular são o íon sódio $\left(\mathrm{Na}^{+}\right)$e o íon cálcio $\left(\mathrm{Ca}^{++}\right)$.

Após o trauma ocorre a abertura dos canais de $\mathrm{Na}^{+}$ voltagem-dependente, o que determina o influxo celular desse íon. Tais canais se abrem em conseqüência de uma despolarização da membrana celular, secundária à depleção dos estoques celulares de adenosina trifosfato (ATP) devido à isquemia celular ${ }^{19}$. O acúmulo intracelular de sódio contribui para surgimento de edema citotóxico, estímulo da ação das fosfolipases e acidose intracelular. Além disso, promove o influxo celular de cálcio e potássio ${ }^{51}$.

A profusão de íons $\mathrm{Ca}^{++}$na célula desencadeia uma cascata de eventos que é caracterizada pela ativação de fosfolipases, proteases, calpaína, plasmalogenases, óxido nítrico sintetase, calcineurinas e endonucleases ${ }^{40}$. Tal situação prejudica a função celular, ocasionando a formação de radicais livres e alterações no DNA celu$\operatorname{lar}^{41}$, o que contribui para a morte da célula. Igualmente é responsável pela degeneração axonal após o trauma em conseqüência da degradação (proteólise) das proteínas neurofilamentares e do citoesqueleto celular ${ }^{46}$ e, outrossim, desencadeia um mecanismo de aumento progressivo do $\mathrm{Ca}^{++}$intracelular (feedback positivo) ${ }^{58}$.

\section{Mecanismos bioquímicos}

Os mecanismos bioquímicos são determinados pela liberação celular de aminoácidos, como o glutamato e o aspartato, em virtude da despolarização da membrana celular. Com isso, ocorre a ativação de receptores glutaminérgicos ionotrópicos (KA, AMPA e NMDA) e metabotrópicos, além da ativação de receptores opiáceos ${ }^{36}$.

Os receptores AMPA e KA, quando ativados, desbloqueiam o receptor NMDA (bloqueio voltagemdependente mediado por magnésio ${ }^{60} \mathrm{e}$ provocam o influxo celular de sódio e potássio, com conseqüente despolarização da membrana e liberação de mais aminoácidos pela célula (feedback positivo). A ativação do receptor NMDA promove influxo celular de cálcio e lesão celular via segundo mensageiro (óxido nítrico) ${ }^{10}$.

Os receptores metabotrópicos, quando de sua ativação, estimulam a fosfolipase $\mathrm{C}$, causando hidrólise do fosfatidil-inositol da membrana plasmática, e aumentam a atividade de todos os outros receptores glutaminérgicos, provocando morte celular por mecanismo de excitotoxicidade ${ }^{1,38}$.

Quanto aos receptores opiáceos pouco se sabe. É conhecido o fato de o trauma medular liberar dinorfina (peptídeo opióide endógeno), que determina ativação de receptores Kappa e de receptores glutaminérgicos $\mathrm{NMDA}^{37,48}$.

\section{Mecanismos inflamatórios}

Os mecanismos inflamatórios envolvem a produção de eicosanóides, a expressão do fator nuclear $\mathrm{kB}$ e a produção de citocinas ${ }^{22,62}$.

O ácido aracdônico, liberado pela lesão da membrana celular, ativa a cicloxigenase e a lipoxigenase, resultando na produção de eicosanóides (prostaglandinas, prostaciclinas, tromboxanas e leucotrienos), que são potentes vasoconstritores e estão envolvidos, como já citados, na fisiopatologia da isquemia medular.

Além disso, tais substâncias determinam o acúmulo local de neutrófilos que causam a liberação de proteases e estão relacionados com a produção de radicais livres. Tal estresse oxidativo está envolvido na gênese da isquemia, além de estimular o acúmulo de mais neutrófilos (feedback positivo) $)^{52,64}$.

$\mathrm{O}$ aumento da expressão do fator nuclear kB (NF$\mathrm{kB})$, responsável pela mediação da resposta inflamatória pós-trauma, determina a produção de prostaglandina sintetase-2, citocinas, moléculas de adesão celular e de óxido nítrico sintetase $\mathrm{e}^{5,6}$. 
O NF-kB também induz a apoptose e a transdução celular anorma ${ }^{30}$.

As citocinas, principalmente o fator de necrose tumoral alfa (TNF- $\alpha$ ), e as interleucinas 1 e 6 (IL-1 e IL-6) potencializam a ação da NF-kB (feedback positivo) e potencializam a microgliose, astrogliose e a morte celular ${ }^{15,31}$

\section{Mecanismos celulares}

Os mecanismos celulares envolvem a produção de radicais livres, a proteína Nogo e a apoptose.

Os radicais livres são produzidos devido a uma falência mitocondrial e causam peroxidação lipídica, responsável por inativação de todas as enzimas fosfolípides dependentes, destruição dos gradientes iônicos celulares, lise da membrana celular e liberação de mais radicais livres (feedback positivo) ${ }^{20}$.

A proteína Nogo é uma proteína derivada da mielina que está presente em grande quantidade no local da lesão medular. Ela é um inibidor do crescimento axonal, impedindo a regeneração do axônio e induzindo à gliose ${ }^{29,47}$.

A apoptose pode ser definida como um processo celular ativo, mediado pela ativação das caspases ${ }^{31,63}$, onde ocorre contração celular com agregação da cromatina, picnose nuclear e morte celular. Tal processo envolve gasto de energia, ao contrário da necrose celular, que é um processo passivo ${ }^{25}$. São os oligodendrócitos os principais acometidos pela apoptose, que também ocorre em axônios com degeneração walleriana, tanto acima como abaixo do local da lesão medular inicial, na área de penumbra isquêmica. A apoptose pode ocorrer de um dia até três semanas após o trauma ${ }^{13}$.

\section{Conclusão}

A lesão medular traumática é um evento que determina inúmeras conseqüências deletérias para o paciente, seus familiares e para a sociedade.

Múltiplos são os fatores envolvidos na sua fisiopatologia e a compreensão desses fatores e das suas interrelações possibilita o surgimento de alvos terapêuticos que certamente, um dia, irão possibilitar o tratamento do paciente com TRM.

Obviamente, é também muito importante investir em campanhas de prevenção da lesão primária, como vem sendo feito por diversos segmentos da sociedade brasileira.

Apesar de a fisiopatologia do TRM ser complexa, é importante que os mecanismos envolvidos na gênese da lesão primária e, especialmente, da lesão secundária permaneçam na memória dos médicos e demais profissionais envolvidos no tratamento dos pacientes com lesão medular. Isso possibilitará, sempre, que novas abordagens terapêuticas sejam consideradas e facilitará o julgamento da aplicabilidade de tratamentos que venham a ser propostos.

Somente assim poderemos mudar os prognósticos neurológico, psicológico e social do paciente com traumatismo raquimedular.

\section{Referências}

1. AGRAWAL SK, THERIAULT E, FEHLINGS MG: Role of group I metabotropic glutamate receptors in traumatic spinal cord white matter injury. J Neurotrauma 15:929-41, 1998.

2. ALLEN AR: Surgery of experimental lesion of spinal cord equivalent to crush injury of fracture dislocation of spinal column. A preliminary report. JAMA 57:878-80, 1911.

3. ALLEN AR: Remarks on the histopathological changes in the spinal cord due to impact. An experimental study. J Nerv Ment Dis 41:141-7, 1941.

4. AMAR AP, LEVY ML: Pathogenesis and pharmacological strategies for mitigating secondary damage in acute spinal cord injury. Neurosurgery 44:1027-39, 1999.

5. BAEUERLE PA: The inducible transcription activator NF-eB: regulation by distinct protein subunits. Biochim Biophys Acta 1072:63-80, 1991.

6. BAEUERLE PA, BALTIMORE D. NF-kappa B: ten years after. Cell 87:13-20, 1996.

7. BOTELHO RV, ABGUSSEN CMB, MACHADO GCFP, ELIAS AJR, BENEDITO-SILVAAA, BITTENCOURT LRA et al.: Epidemiologia do trauma raquimedular cervical na zona norte da cidade de São Paulo. Arq Bras Neurocir 20(3/4):64-76, 2001.

8. BURNEY RE, MAIO RF, MAYNARD F, KARUNAS R: Incidence, characteristics and outcome of spinal cord injury at trauma centers in North America. Arch Surg 128:596-99, 1993.

9. CUNHA FM, MENEZES CM, GUIMARÃES EP: Lesões traumáticas da coluna torácica e lombar. Rev Bras Ortop 35(1/2):17-22, 2000

10. DAWSON VL, DAWSON TM, LONDON ED, BREDT DS, SNYDER SH: Nitric oxide mediates glutamate neurotoxicity in primary cortical cultures. Proc Natl Acad Sci USA 88:6368-71, 1991.

11. DOHRMANN GJ, ALLEN WE: Microcirculation of traumatized spinal cord. A correlation of microangiography and blood flow patterns in transitory and permanent paraplegia. J Trauma 15:1003-13, 1975.

12. ELSBERG CA: The Edwin Smith surgical papyrus and the diagnosis and treatment of injuries to the skull and spine 5000 years ago. Ann Med Hist 3:271-9, 1931.

13. EMERY E, ALDANA P, BUNGE MB et al.: Apoptosis after traumatic human spinal cord injury. J Neurosurg 89:911-20, 1998.

14. FAWCETT JW, CURT A, STEEVES JD, COLEMAN WP, TUSZYNSKI MH, LAMMERTSE D et al.: Guidelines for the conduct of clinical trials for spinal cord injury as developed by the ICCP panel: spontaneous recovery after spinal cord 
injury and statistical power needed for therapeutic clinical trials. Spinal Cord 45:190-205, 2007.

15. FEUERSTEIN GZ, LIU T, BARONE FC: Cytokines, inflammation and brain injury: role of tumor necrosis factor-alpha. Cerebrovasc Brain Metab Rev 6:341-60, 1994.

16. FREITAS PEP: Traumatismos raquemedulares agudos: estudo epidemiológico de cem casos consecutivos. J Bras Neurocir 2:1-10, 1990.

17. FRIGON A, ROSSIGNOL S: Functional plasticity following spinal cord lesions. Prog Brain Res 157:231-60, 2006.

18. GUHA A, TATOR CH: Acute cardiovascular effects of experimental spinal cord injury. J Trauma 28:481-90, 1988.

19. HAIGNEY MC, LAKATTA EG, STERN MD, SILVERMAN HS: Sodium channel blockade reduces hypoxic sodium loading and sodium-dependent calcium loading. Circulation 90:391-9, 1994

20. HALL ED, BRAUGHLER JM: Free radicals in CNS injury. Res Publ Assoc Res Nerv Ment Dis 71:81-105, 1993.

21. HARROP JS, SHARAN A, RATLIFF J: Central cord injury: pathophysiology, management, and outcomes. J Spine 6(6 Suppl):S198-206, 2006.

22. HAUSMANN ON: Post-traumatic inflammation following spinal cord injury. Spinal Cord 41:369-78, 2003.

23. HEINEMANN AW, YARKONY GM, ROTH EJ, LOVELL L, HAMILTON B, GINSBURG K et al.: Functional outcome following spinal cord injury. A comparison of specialized spinal cord injury center vs general hospital short-term care. Arch Neurol 46:1098-102, 1989.

24. HO CH, WUERMSER LA, PRIEBE MM, CHIODO AE, SCELZAWM, KIRSHBLUM SC: Spinal cord injury medicine. 1. Epidemiology and classification. Arch Phys Med Rehabil 88(3 Suppl 1):S49-54, 2007.

25. HOCKENBERY D: Defining apoptosis. Am J Pathol 146:16-9, 1995

26. HSU CY, HOGAN EL, GADSEN RH, SPICER KM, SHI MP, COX RD: Vascular permeability in experimental spinal cord injury. J Neurol Sci 70:275-82, 1985.

27. HUGHES JT: The Edwin Smith Surgical Papyrus: an analysis of the first case reports of spinal cord injuries. Paraplegia 26:71-82, 1988.

28. HULSEBOSCH CE: Recent advances in pathophysiology and treatment of spinal cord injury. Adv Physiol Educ 26(1-4): 238-55, 2002.

29. JACOBS WB, FEHLINGS MG: The molecular basis of neural regeneration. Neurosurgery 53:943-8, 2003.

30. KALTSCHMIDT B, BAEUERLE PA, KALTSCHMIDT C: Potential involvement of the transcription factor NF-kappa B in neurological disorders. Mol Aspects Med 14:171-90, 1993.

31. KEANE RW, DAVIS AR, DIETRICH WD: Inflammatory and apoptotic signaling after spinal cord injury. J Neurotrauma 23(3-4):335-44, 2006.

32. KOBRINE AI, DOYLE TF, MARTINS AN: Local spinal cord blood flow in experimental traumatic myelopathy. J Neurosurg 42:144-9, 1975.

33. KRAUS JF: Injury to the head and spinal cord. The epidemiological relevance of the medical literature published from 1960 to 1978. J Neurosurg 53(Suppl):S3-10, 1980.

34. KRAUS JF, FRANTI CE, RIGGINS RS, RICHARDS D, BORHANI NO: Incidence of traumatic spinal cord lesions. J Chronic Dis 28:471-92, 1975.

35. KURTZKE JF: Epidemiology of spinal cord injury. Exp Neurol 48(3 Pt 2):163-236, 1975

36. LODGE D, COLLINGRIDGE G: Les agents provocateurs: a series on the pharmacology of excitatory amino acids. Trends Pharmacol Sci 11:22-4, 1990.
37. LONG JB, MARTINEZ-ARIZALA A, PETRAS JM, HOLADAY JW: Endogenous opioids in spinal cord injury: a critical evaluation. Cent Nerv Syst Trauma 3:295-315, 1986.

38. MANEV H, FAVARON M, GUIDOTTI A, COSTA E: Delayed increase of $\mathrm{Ca} 2+$ influx elicited by glutamate: role in neuronal death. Mol Pharmacol 36:106-12, 1989.

39. NORENBERG MD, SMITH J, MARCILLO A: The pathology of human spinal cord injury: defining the problems. J Neurotrauma 21:429-40, 2004.

40. RAY SK, HOGAN EL, BANIK NL: Calpain in the pathophysiology of spinal cord injury: neuroprotection with calpain inhibitors. Brain Res Rev 42:169-85, 2003.

41. RAY SK, SHIELDS DC, SAIDO TC, MATZELLE DC, WILFORD GG, HOGAN EL et al.: Calpain activity and translational expression increased in spinal cord injury. Brain Res 816:375-80, 1999.

42. RICHARDSON PM, MCGUINNESS UM, AGUAYO AJ: Regeneration following sciatic nerve grafting to the rat spinal cord. Can J Neurol Sci 6:395, 1979.

43. RICHARDSON PM, MCGUINNESS UM, AGUAYO AJ: AXons from CNS neurons regenerate into PNS grafts. Nature 284:264-5, 1980.

44. RIDDOCH G: The practical significance of the mass reflex in the treatment of injuries of the spinal cord. Lancet 2:839-41, 1917.

45. SCHMAUS H: Beiträge zur pathologischen anatomie der rückenmarkserschütterung. Virchows Arch 122:470-95, 1980. Apud Tator CH, Benzel EC (ed): Comtemporary Management of Spinal Cord Injury: From Impact to Rehabilitation. AANS Publications Committee, 2000.

46. SCHUMACHER PA, EUBANKS JH, FEHLINGS MG: Increased calpain I-mediated proteolysis, and preferential loss of dephosphorylated NF200, following traumatic spinal cord injury. Neuroscience 91:733-44, 1999.

47. SCHWEIGREITER R, BANDTLOW CE: Nogo in the injured spinal cord. J Neurotrauma 23(3-4):384-96, 2006.

48. SHUKLA VK, LEMAIRE S: Central non-opiod physiological and pathophysiological effects of dynorphin $A$ and related peptides. J Psychiatry Neurosci 17:106-19, 1992.

49. SONNTAG VK, DOUGLAS RA: Management of spinal cord trauma. Neurosurg Clin N Am 1:729-50, 1990.

50. STRIPLING TE: The cost of economic consequences of traumatic spinal cord injury. Paraplegia News 8:50-4, 1990.

51. STYS PK, WAXMAN SG, RANSOM BR: Ionic mechanisms of anoxic injury in mammalian CNS white matter: role of $\mathrm{Na}+$ channels and the $\mathrm{Na}+-\mathrm{Ca} 2+$ exchanger. J Neurosci 12:430-9, 1992.

52. TAOKAY, OKAJIMAK, UCHIBAM, MURAKAMI K, HARADA $\mathrm{N}, \mathrm{JOHNO} \mathrm{M}$ et al.: Role of Neutrophils in spinal cord injury in the rat. Neuroscience 79:1177-82, 1997.

53. TARLOV IM, KLINGER H: Spinal cord compression studies II. Time limits for recovery after acute compression in dogs. AMA Arch Neurol Psychiatry 71:271-90, 1954.

54. TATOR CH, FEHLINGS MG: Review of secondary injury theory of acute spinal cord trauma with emphasis on vascular mechanisms. J Neurosurg 75:15-26, 1991.

55. TATOR CH, DUNCAN EG, EDMONDS VE, LAPCZAK LI, ANDREWS DF: Complications and costs of management of acute spinal cord injury. Paraplegia 31:700-14, 1993.

56. TATOR $\mathrm{CH}$ : Update on pathophysiology and pathology of acute spinal cord inury. Brain Pathol 5:407-13, 1995.

57. TATOR CH, KOYANAGI I: Vascular mechanisms in the pathophysiology of human spinal cord injury. J Neurosurg 86:483-92, 1997. 
58. TYMIANSKI M, TATOR CH: Normal and abnormal calcium homeostasis in neurons: a basis for the pathophysiology of traumatic and ischemic central nervous system injury. Neurosurgery 38:1176-95, 1996.

59. WINSLOW C, ROZOVSKY J: Effect of spinal cord injury on the respiratory system. Am J Phys Med Rehabil 82:803-14, 2003.

60. WRATHALL JR, CHOINIERE D, TENG YD: Dose-dependent reduction of tissue loss and functional impairment after spinal cord trauma with the AMPA/kainate antagonist NBQX. J Neurosci 14(11 Pt 1):6598-607, 1994.

61. YASHON D, BINGHAM JR WG, FADDOUL EM, HUNT WE: Edema of spinal cord following experimental impact trauma. J Neurosurg 38:693-7, 1973.

62. YOUNG W: Secondary injury mechanisms in acute spinal cord injury. J Emerg Med 11 (Suppl 1):S13-22, 1993.
63. YUAN J, HORVITZ HR: A first insight into the molecular mechanisms of apoptosis. Cell 116(Suppl 2):S53-56, 2004.

64. XU JA, HSU CY, LIU TH, HOGAN EL, PEROT JR PL, TAI HH: Leukotriene $B 4$ release and polymorphonuclear cell infiltration in spinal cord injury. J Neurochem 55:907-12, 1990.

Original recebido em maio de 2007

Aceito para publicação em março de 2008

\section{Endereço para correspondência}

Sergio Henrique do Amaral

Rua Afonso Braz, 473, cj. 36

04511-011 - São Paulo, SP, Brasil

E-mail:drshamaral@uol.com.br 